Are affective factors related to individual differences in facial expression recognition?

Sarah AH Alharbi ${ }^{1,2}$, Katherine Button ${ }^{3}$, Amy Bagshaw $^{4}$, Lingshan Zhang ${ }^{1}$, Kieran J O'Shea ${ }^{1}$, Vanessa Fasolt ${ }^{1}$, Anthony $\mathrm{J} \mathrm{Lee}^{5}$, Lisa M DeBruine ${ }^{1}$, Benedict $\mathrm{C}$ Jones $^{1}$

1. Institute of Neuroscience \& Psychology, University of Glasgow, Scotland, UK.

2. Department of Psychology, Taibah University, Medina, KSA.

3. Department of Psychology, University of Bath, England, UK.

4. School of Life Sciences, University of Glasgow, Scotland, UK.

5. Division of Psychology, University of Stirling, Scotland, UK.

Corresponding author: Benedict Jones (ben.jones@glasgow.ac.uk)

We thank Jeffrey Girard for helpful feedback on an earlier draft. 


\title{
Are affective factors related to individual differences in facial expression recognition?
}

\begin{abstract}
Evidence that affective factors (e.g., anxiety, depression, affect) are significantly related to individual differences in emotion recognition is mixed. Palermo et al. (2018 Journal of Experimental Psychology: Human Perception and Performance) recently reported that individuals who scored lower in anxiety performed significantly better on two measures of facial-expression recognition (emotion-matching and emotion-labeling tasks), but not a third measure (the Multimodal Emotion Recognition Test). By contrast, facialexpression recognition was not significantly correlated with measures of depression, positive or negative affect, empathy, or autistic-like traits. Because the range of affective factors considered in this study and its use of multiple expression-recognition tasks mean that it is a relatively comprehensive investigation of the role of affective factors in facial expression recognition, we propose to carry out a direct replication.
\end{abstract}

\section{Introduction}

Facial expression recognition plays an important role in social interaction. Although it is widely acknowledged that substantial individual differences in facial expression recognition exist, the factors that underpin these individual differences are poorly understood (Palermo et al., 2018). Many studies that have investigated this issue have focused on the role of affective factors, such as anxiety, depression, mood, and empathy.

Evidence from studies investigating the relationship between affective factors and facial expression recognition has been mixed. For example, while studies of clinical samples have found that anxious or depressed people show impaired facial expression recognition (e.g., Bistricky et al., 2011; Demenescu et al., 2010), some studies of non-clinical samples have not observed significant correlations between facial expression recognition and anxiety or 
depression (e.g., Surcinelli et al., 2006). Similarly, while some studies have reported that people who score higher on measures of empathy or lower on measures of autistic-like traits perform better on facial expression recognition tasks (e.g., Lewis et al., 2016; Poljac et al., 2013), other studies have not replicated these results (e.g., Palermo et al., 2018).

Interpreting the mixed results for affective factors and facial expression recognition described above is complicated because different studies have investigated different affective factors and/or used different methods to assess facial expression recognition. Direct replications (i.e., studies using the same measures as the original work) are one way to address this difficulty because they allow for more direct comparison of results across studies (Simons, 2014).

In light of the above, we propose to directly replicate one recent study of the possible link between affective factors and facial expression recognition (Palermo et al., 2018). We chose this particular study to replicate because it considered a relatively broad range of affective factors (various measures of anxiety, depression, mood, and empathy) and showed consistent results across two recently developed comprehensive facial expression recognition tasks (the emotion-matching and emotion-labeling tasks developed and described in Palermo et al., 2013). We also chose Palermo et al. (2018) for our direct replication because, despite these methodological strengths, the significant relationships between affective factors and facial expression recognition would not have been significant if corrected for multiple comparisons. This pattern of results suggests that the correlations between anxiety and facial expression recognition may not necessarily be robust.

Palermo et al. (2018) reported that participants' ( $N=63)$ scores on the anxiety scale of the Depression Anxiety and Stress Scales (DASS) were negatively correlated with their performance on Palermo et al's (2013) emotion-matching $(r=-.287, p=.023)$ and emotion-labeling $(r=-.255, p=.044)$ tasks. By contrast 
with their results for anxiety, participants' performance on neither of these emotion-recognition tasks was significantly correlated with their scores on questionnaires measuring a range of other affective factors (empathy, depression, or mood). Performance on a third emotion recognition test (Bänziger et al's 2009 Multimodal Emotion Recognition Test) was not significantly correlated with any of the affective factors. Based on these results, Palermo et al. (2018) concluded that anxiety is the critical affective factor for individual differences in facial expression recognition.

Following Palermo et al's (2018) results, we will test four specific hypotheses:

Hypothesis 1. Scores on the anxiety scale of the Depression Anxiety and Stress Scales (DASS) will be significantly negatively correlated with performance on the emotion-matching task.

Hypothesis 2. Scores on the anxiety scale of the Depression Anxiety and Stress Scales (DASS) will be significantly negatively correlated with performance on the emotion-labeling task.

Hypothesis 3. Performance on neither the emotion-matching nor emotionlabeling tasks will be significantly correlated with scores on the depression scale of the Depression Anxiety and Stress Scales (DASS), the positive affect scale of the Positive and Negative Affect Schedule (PANAS), the negative affect scale of the Positive and Negative Affect Schedule (PANAS), scores on the Autism Quotient (AQ), scores on the Empathy Quotient (EQ), scores on the affective component of the Basic Empathy Scale (BES), or scores on the cognitive component of the Basic Empathy Scale (BES).

Hypothesis 4. Performance on Bänziger et al's (2009) Multimodal Emotion Recognition Test will not be significantly correlated with scores on any of the affective factors.

\section{Methods}

\section{Ethics}


All aspects of this project have been approved by the University of Glasgow College of Science and Engineering Ethics Committee (application number 300180047).

\section{Participants and justification of sample size}

Palermo et al. (2018) tested 63 university students (55\% women, 45\% men). Simonsohn (2015) recommends that sample sizes in replication studies be approximately two and a half times the sample size of the original study. Consequently, we will test 160 University of Glasgow students between the ages of 16 and 45 years with a similar sex ratio to Palermo et al's (2018) original study.

\section{Emotion-matching task}

We will use the same emotion-matching task as Palermo et al. (2018). This is the 100-item emotional masking task originally developed by Palermo et al. (2013). Images of three different individuals (matched for sex) are presented on each trial. Two of the images (the distractor images) are shown with the same emotional expression (e.g., anger). The other image (the target image) is shown with a different emotional expression (e.g., disgust), making it the 'odd one out'. Target and distractor emotions are paired to be maximally confusable accordingly to previously published data (Young et al., 2002). Participants use numbered keys to indicate whether face 1, face 2, or face 3 is displaying the 'odd-one-out' emotion. Participants can respond either while the faces are presented onscreen $(4500 \mathrm{~ms})$ or any time up to $7000 \mathrm{~ms}$ time after the faces are no longer presented onscreen. The 100 trials are presented in the same order for each participant and are preceded by eight practice trials. The stimuli, target-distractor pairings, and trial order we will use are identical to those in Palermo et al. (2013) and described in their supplemental materials. Performance on this task is indicated by the percentage of trials on which a participant correctly identifies the target face. An example trial from the emotion-matching task is shown in Figure 1. We have obtained the code and stimuli for this task from the corresponding author of Palermo et al. (2018), allowing us to precisely replicate this task. Stimuli are 
from the Karolinska Directed Emotional Faces image database (Lundqvist et al., 1998), will be shown in color on an iMAC12.1, at $1686 \times 762$ pixels.

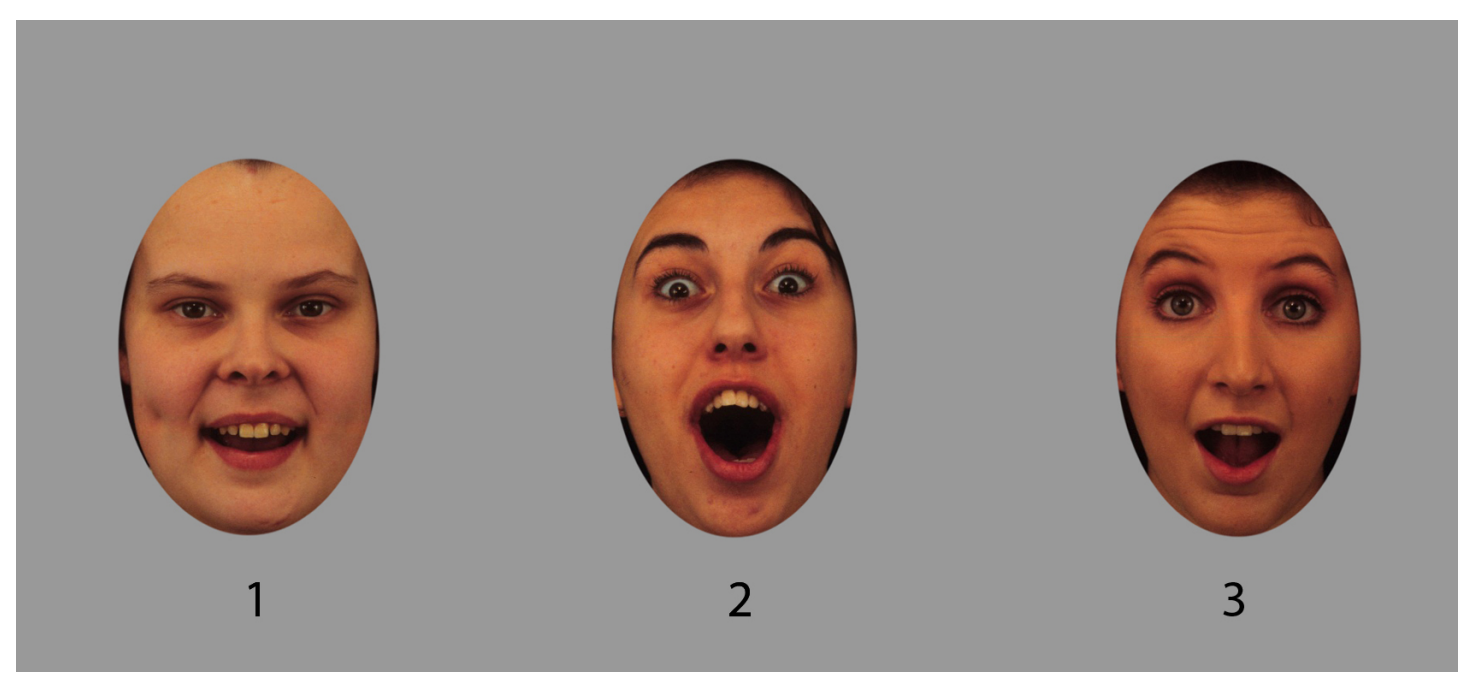

Figure 1. An example trial from the emotion-matching task. Participants are instructed to indicate which face is showing a different emotional expression to the other two faces (i.e., which emotion is the 'odd-one-out'). The correct answer on the trial shown is face 1.

\section{Emotion-labeling task}

We will use the same emotion-matching task as Palermo et al. (2018). This is the 100-item emotion-labeling task originally developed by Palermo et al. (2013), but with two modifications (presentation time for each face reduced from $1000 \mathrm{~ms}$ to $400 \mathrm{~ms}$ and the number of facial expressions in each emotion category being the same). Both of these modifications to the task described in Palermo et al. (2013) were also made in Palermo et al. (2018). Each face is individually presented on screen. Participants use a computer mouse to select the appropriate emotion label from a set of six labels presented underneath the face (anger, disgust, fear, surprise, sadness, happiness). Responses can be made while the face is presented $(400 \mathrm{~ms})$ or up to $7000 \mathrm{~ms}$ after the face is no longer presented onscreen. The stimuli and trial order we will use are identical to those used in Palermo et al. (2018) and described in an email provided by the corresponding author of the Palermo et al. (2018) paper. Performance on this task is indicated by the percentage of trials on which the participant correctly labels the facial expression. An example trial from the emotion-labeling task is shown in Figure 2. We have obtained the code and stimuli for this task from the corresponding author of Palermo et al. (2018), allowing us to precisely replicate this task. Stimuli are from the Karolinska 
Directed Emotional Faces image database (Lundqvist et al., 1998), will be shown in color on a iMAC12.1 monitor, at $737 \times 737$ pixels.

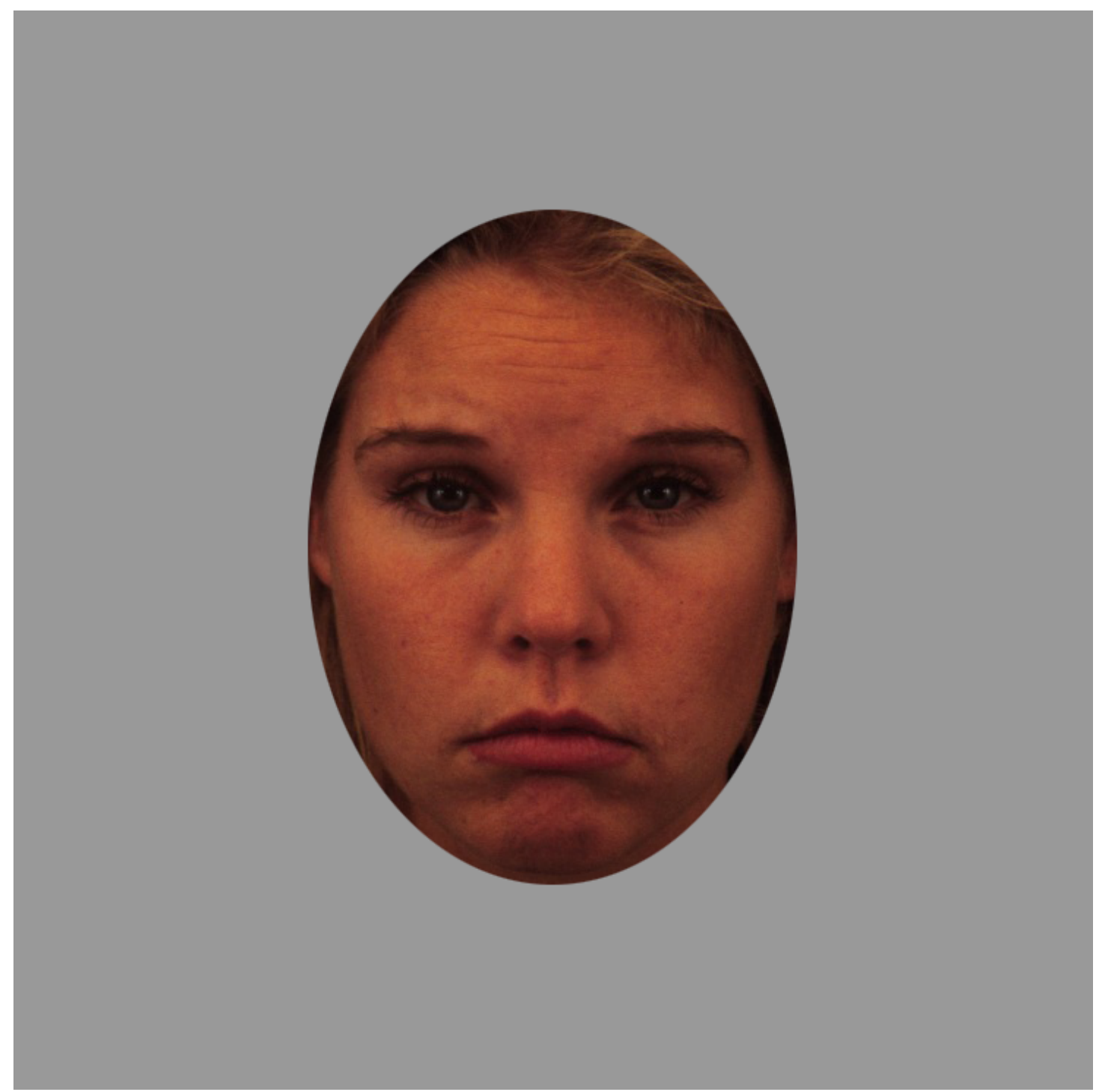

Figure 2. An example trial from the emotion-labeling task. Participants are instructed to select the appropriate emotion label from a set of six labels presented underneath the face (anger, disgust, fear, surprise, sadness, happiness). The correct answer on the trial shown is sadness.

\section{Multimodal Emotion Recognition Test}

This test, described in full in Bänziger et al. (2009), is an online test administered via the Swiss Centre for Affective Sciences webpage. The task consists of 30 video clips of actors (three for each of 10 emotions: irritation, anger, anxiety, fear, happiness, elated joy, disgust, contempt, sadness, despair) that are presented in four modalities (still picture, video only, audio only, audio with video), yielding a total of 120 items. After viewing each clip, 
participants are asked to label the emotion shown in the clip. We will use the English-language version of the task for our replication.

\section{Affective factors questionnaires}

Each participant will complete the same affective factor questionnaires used by Palemro et al. (2018). These are Lovibond and Lovibond's (1995)

Depression Anxiety and Stress Scales (DASS), Watson et al's (1988) Positive and Negative Affect Schedule (PANAS), Baron-Cohen et al's (2001) Autism Quotient (AQ), Baron-Cohen and Wheelwright's (2004) Empathy Quotient (EQ), and Jolliffe and Farrington's (2006) Basic Empathy Scale (BES).

\section{Procedure}

As in Palermo et al. (2018), the DASS and PANAS will be administered before the emotion-labeling and emotion-matching tasks. The other affective questionnaires will be administered after the emotion-labeling and emotionmatching tasks. Following Palermo et al. (2018), we will run the emotionmatching task before the emotion-labeling task and run both of these tasks before the Multimodal Emotion Recognition Test.

All subscale and component scores tested by Palermo et al. (2018) will be calculated following the instructions for these questionnaires. The scales we will investigate are the anxiety scale of the DASS, the depression scale of the DASS, the positive affect scale of the PANAS, the negative affect scale of the PANAS, the $A Q$, the EQ scores, scores on the affective component of the BES, and scores on the cognitive component of the BES. These are the same scales employed by Palermo et al. (2018). Although Palermo et al. (2018) do not report the reliability of these measures, we will report Cronbach's alpha for each scale.

\section{Data exclusions and data quality checks}

Outliers (scores on a measure that are more than 3 standard deviations from the mean score for that measure) will be adjusted to score one point higher than the closest non-outlier score (following Palermo et al., 2018). As a positive control, participants scoring lower than chance on either of the 
expression recognition tasks will be excluded from all analyses. No other exclusions or data manipulation will be carried out.

\section{Analysis plan}

Hypothesis 1. Scores on the anxiety scale of the Depression Anxiety and Stress Scales (DASS) will be significantly negatively correlated with performance on the emotion-matching task.

As in Palermo et al. (2018), we will test Hypothesis 1 by calculating Pearson's product-moment correlation coefficient for the relationship between scores on the anxiety scale of the Depression Anxiety and Stress Scales (DASS) and performance on the emotion-matching task. Our sample size has $80 \%$ power to detect effects as small as $|r|=.219$ at the $5 \%$ significance level. Analysis code for Hypothesis 1 is publicly available at https://osf.io/kexhr/ and in our supplemental materials.

Hypothesis 2. Scores on the anxiety scale of the Depression Anxiety and Stress Scales (DASS) will be significantly negatively correlated with performance on the emotion-labeling task.

As in Palermo et al. (2018), we will test Hypothesis 2 by calculating Pearson's product-moment correlation coefficient for the relationship between scores on the anxiety scale of the emotion-labeling task. Depression Anxiety and Stress Scales (DASS) and performance on the. Our sample size has $80 \%$ power to detect effects as small as $|r|=.219$ at the $5 \%$ significance level. Analysis code for Hypothesis 2 is publicly available at https://osf.io/kexhr/ and in our supplemental materials.

Hypothesis 3. Performance on neither the emotion-matching nor emotionlabeling tasks will be significantly correlated with scores on the depression scale of the DASS, the positive affect scale of the PANAS, the negative affect scale of the PANAS, AQ scores, EQ scores, scores on the affective component of the BES, or scores on the cognitive component of the BES. 
As in Palermo et al. (2018), we will test Hypothesis 3 by calculating the Pearson's product-moment correlation coefficients for the relationships between the emotion-matching and emotion-labeling tasks and scores on the depression scale of the DASS, the positive affect scale of the PANAS, the negative affect scale of the PANAS, AQ scores, EQ scores, scores on the affective component of the BES, and scores on the cognitive component of the BES. Our sample size has $80 \%$ power to detect effects as small as $|r|=$ .219 at the $5 \%$ significance level. Analysis code for Hypothesis 3 is publicly available at https://osf.io/kexhr/ and in our supplemental materials.

If, by contrast with Palermo et al. (2018), other questionnaires also predict performance on emotion-recognition tasks, we will use Steiger's test for comparing elements of a correlation matrix (Steiger, 1980) to test whether scores on the anxiety scale of the DASS are a significantly better predictor of performance on the emotion-recognition tasks than are scores on those other questionnaires.

Hypothesis 4. Performance on Bänziger et al's (2009) Multimodal Emotion Recognition Test will not be significantly correlated with scores on any of the affective factors.

As in Palermo et al. (2018), we will test Hypothesis 4 by calculating the Pearson's product-moment correlation coefficients for the relationships between the Multimodal Emotion Recognition Test and scores on the depression scale of the DASS, the positive affect scale of the PANAS, the negative affect scale of the PANAS, AQ scores, EQ scores, scores on the affective component of the BES, and scores on the cognitive component of the BES. Our sample size has $80 \%$ power to detect effects as small as $|r|=$ .219 at the $5 \%$ significance level. Analysis code for Hypothesis 4 is publicly available at https://osf.io/kexhr/ and in our supplemental materials.

\section{Robustness checks}

As in Palermo et al. (2018), we will repeat each analysis using partial correlations to control for possible effects of participant sex, participant age, 
and both participant sex and participant age simultaneously. Analysis code for these robustness checks is publicly available at https://osf.io/kexhr/ and in our supplemental materials.

Following Palermo et al. (2018), we will also test the correlation between each of the affective factors measures and the first component produced by principal component analysis of scores on the three emotion recognition tasks. Scores on this component were significantly correlated with anxiety, but no other affective factors, in Palermo et al. (2018).

We will also conduct additional robustness checks restricting the sample to only those participants who scored within the maximum and minimum values for each measure as reported in Table 1 of Palermo et al. (2018).

\section{Exploratory analyses of social anxiety}

Although data on social anxiety specifically were not collected by Palermo et al. (2018), some researchers have suggested that because of fears concerning negative evaluation, social anxiety may be a key correlate of individual differences in emotion recognition (e.g., Rapee \& Heimberg, 1997; Hirsch \& Clark, 2004). Consequently, we will repeat the analyses described in Hypotheses 1, 2, and 4 (and the related robustness checks) using scores on the Brief Fear of Negative Evaluation Scale (BFNE; Leary, 1983) and the 6item versions of the Social Interaction Anxiety Scale (SIAS) and Social Phobia Scale (SPS) developed by Lorna et al. (2012). So as to not interfere with our replication of Palermo et al's (2018) study, these questionnaires will be administered in a fully randomized order at the very end of the study.

\section{Open data statement}

All data and analysis code will be made publicly available on the Open Science Framework at the point of publication.

\section{References}


Bänziger, T., Grandjean, D., \& Scherer, K. R. (2009). Emotion recognition from expressions in face, voice, and body: the Multimodal Emotion Recognition Test (MERT). Emotion, 9(5), 691-704.

Baron-Cohen, S., \& Wheelwright, S. (2004). The empathy quotient: an investigation of adults with Asperger syndrome or high functioning autism, and normal sex differences. Journal of Autism and Developmental Disorders, 34(2), 163-175.

Baron-Cohen, S., Wheelwright, S., Skinner, R., Martin, J., \& Clubley, E. (2001). The autism-spectrum quotient (AQ): Evidence from Asperger syndrome/high-functioning autism, males and females, scientists and mathematicians. Journal of Autism and Developmental Disorders, 31(1), 5-17.

Bistricky, S. L., Ingram, R. E., \& Atchley, R. A. (2011). Facial affect processing and depression susceptibility: Cognitive biases and cognitive neuroscience. Psychological Bulletin, 137(6), 998-1028.

Demenescu, L. R., Kortekaas, R., den Boer, J. A., \& Aleman, A. (2010). Impaired attribution of emotion to facial expressions in anxiety and major depression. PLoS ONE, 5(12), e15058.

Jolliffe, D., \& Farrington, D. P. (2006). Development and validation of the Basic Empathy Scale. Journal of Adolescence, 29(4), 589-611.

Hirsch, C.R., Clark, D.M. (2004). Information-processing bias in social phobia. Clinical Psychology Review 24,799-825.

Lewis, G. J., Lefevre, C. E., \& Young, A. W. (2016). Functional architecture of visual emotion recognition ability: A Latent Variable Approach. Journal of Experimental Psychology: General, 145(5), 589-602.

Lovibond, S.H. \& Lovibond, P.F. (1995). Manual for the Depression Anxiety Stress Scales. (2nd. Ed.) Sydney, Australia: Psychology Foundation.

Lundqvist, D., Flykt, A., \& Öhman, A. (1998). The Karolinska Directed Emotional Faces - KDEF, CD ROM from Department of Clinical Neuroscience, Psychology section, Karolinska Institutet, ISBN 91-6307164-9.

Palermo, R., Jeffery, L., Lewandowsky, J., Fiorentini, C., Irons, J. L., Dawel, A., ... \& Rhodes, G. (2018). Adaptive face coding contributes to 
individual differences in facial expression recognition independently of affective factors. Journal of Experimental Psychology: Human Perception and Performance, 44(4), 503-517.

Palermo, R., O'Connor, K. B., Davis, J. M., Irons, J., \& McKone, E. (2013). New tests to measure individual differences in matching and labelling facial expressions of emotion, and their association with ability to recognise vocal emotions and facial identity. PLoS ONE, 8(6), e68126.

Poljac, E., Poljac, E., \& Wagemans, J. (2013). Reduced accuracy and sensitivity in the perception of emotional facial expressions in individuals with high autism spectrum traits. Autism, 17(6), 668-680.

Rapee, R.M., Heimberg, R.G. (1997). A cognitive-behavioral model of anxiety in social phobia. Behaviour Research and Therapy 35, 741-756.

Simonsohn, U. (2015). Small telescopes: Detectability and the evaluation of replication results. Psychological science, 26(5), 559-569.

Simons, D. J. (2014). The value of direct replication. Perspectives on Psychological Science, 9(1), 76-80.

Steiger, J. H. (1980). Tests for comparing elements of a correlation matrix. Psychological Bulletin, 87, 245-251.

Surcinelli, P., Codispoti, M., Montebarocci, O., Rossi, N., \& Baldaro, B. (2006). Facial emotion recognition in trait anxiety. Journal of Anxiety Disorders, 20(1), 110-117.

Watson, D., Clark, L. A., \& Tellegen, A. (1988). Development and validation of brief measures of positive and negative affect: the PANAS scales. Journal of Personality and Social Psychology, 54(6), 1063-1070.

Young, A.W., Perrett, D.I., Calder, A.J., Sprengelmeyer, R., \& Ekman, P. (2002) Facial expressions of emotion: Stimuli and tests (FEEST). Bury St. Edmunds: Thames Valley Test Company. 


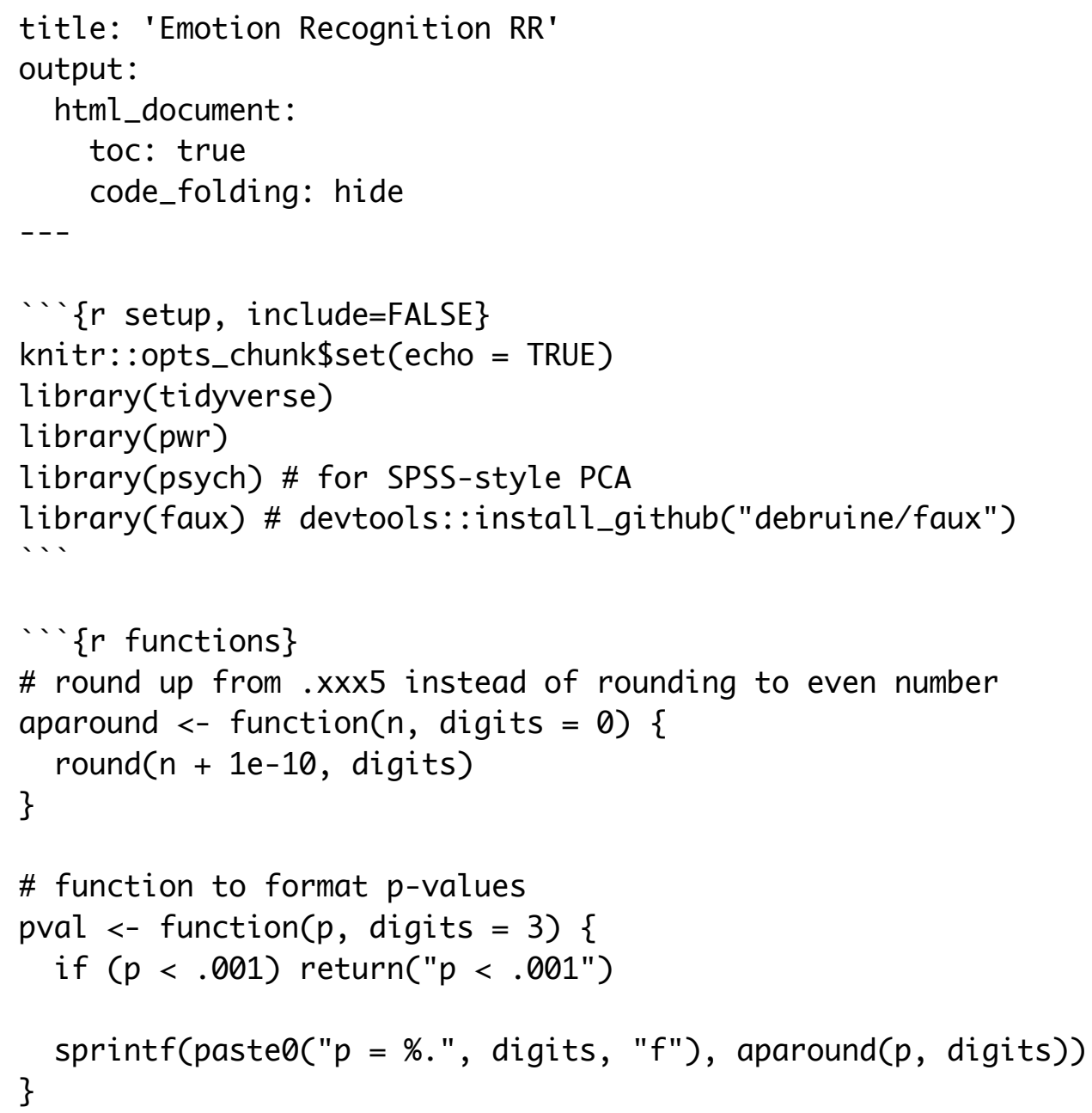

Sample size is set at $\$ n=160 \$$, following the small telescopes approach, which sets replication sample size at 2.5 times the original sample size $(\$ n=63 \$)$. With $\$ a$ pha $=.05 \$$, this design has $80 \%$ power for two-tailed tests to detect correlations as small as $\$|r|={ }^{`} r$ smallest_effect $\$$.

\#\# Analysis

\#\#\# Simulate data 
Descriptive data are taken from from Palermo et al (2018) with correlation structure from correlations reported in Palermo et al. (2018) or set to $r=$ 0.5 for all depression/anxiety measures (as unreported in Palermo).

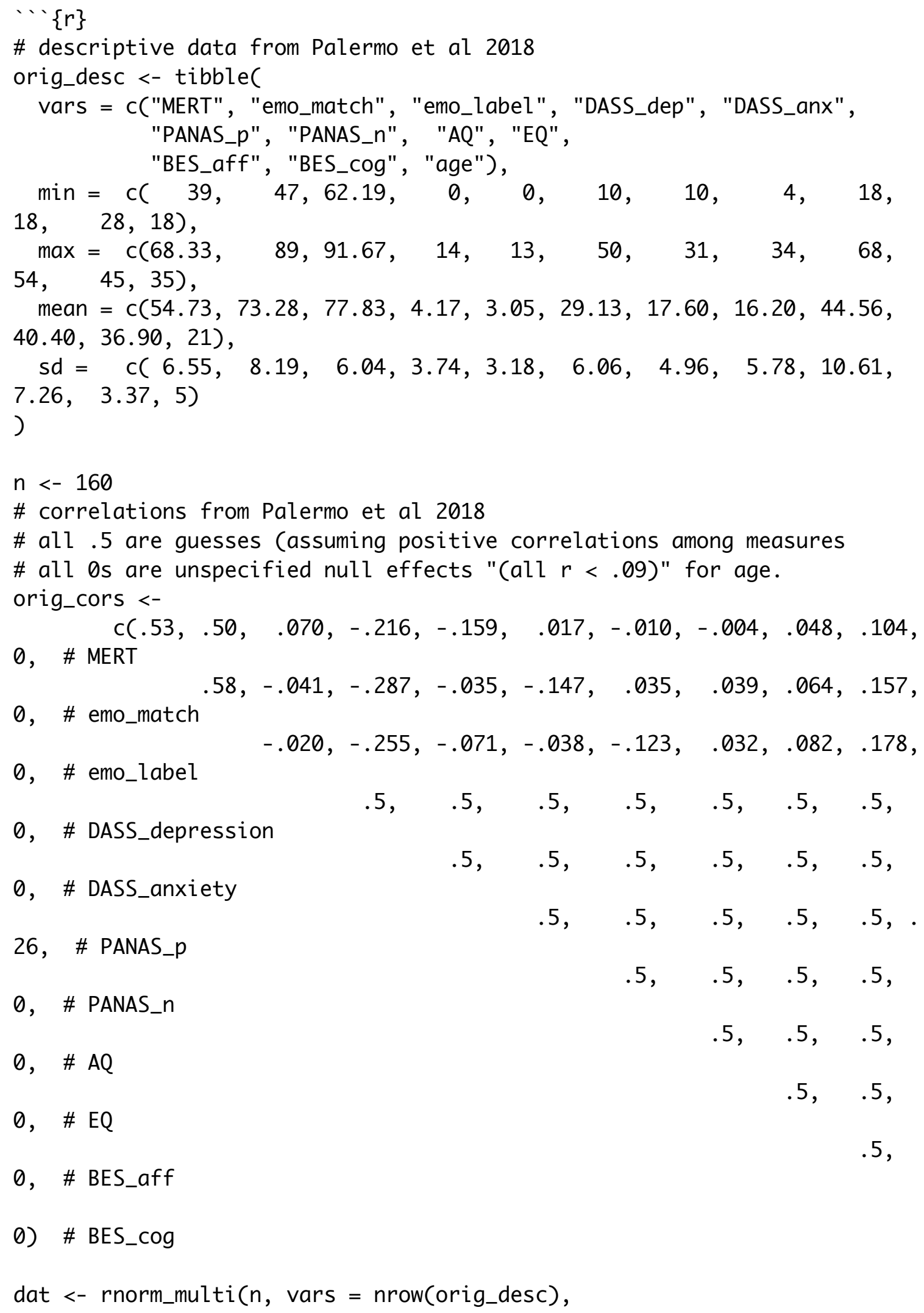




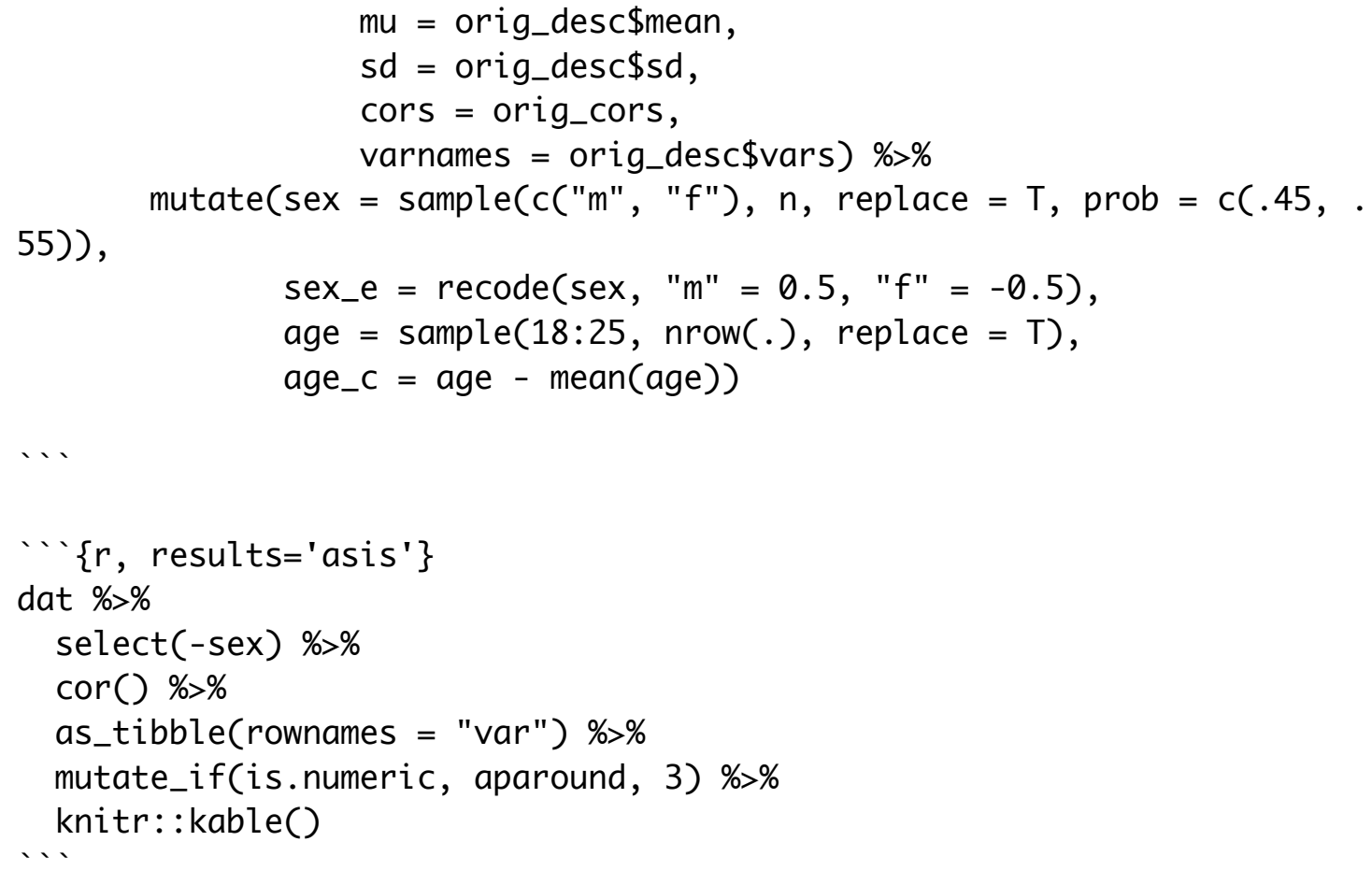

\#\#\# Hypothesis 1

$\cdots\{r \mathrm{H} 1\}$

$\mathrm{H} 1<-$ cor.test(dat\$DASS_anx, dat\$emo_match) $\%>\%$ broom: :tidy() \% \%

mutate_if(is.numeric, aparound, 3)

Scores on the emotion matching task and the DASS anxiety subscale were correlated with $r={ }^{`} r$ H1\$estimate`; 95\% CI $=$ [` $r$ H1\$conf. low`, ' $r$ H1\$conf.high`], ' $r$ pval(H1\$p.value)`.

\#\#\# Hypothesis 2

$\cdots\{r \mathrm{H} 2\}$

$\mathrm{H} 2$ <- cor.test(dat\$DASS_anx, dat\$emo_label) \%>\% broom: :tidy() \%>\%

mutate_if(is.numeric, aparound, 3)

Scores on the emotion labeling task and the DASS anxiety subscale were correlated with $r={ }^{\prime} r$ H2\$estimate`; 95\% CI $=$ [` $r$ H2\$conf. Low', ' $r$ H2\$conf.high'], ' $r$ pval(H2\$p.value)`.

\#\#\# Hypothesis 3

․ $\{r$ H3, results='asis' $\}$ 


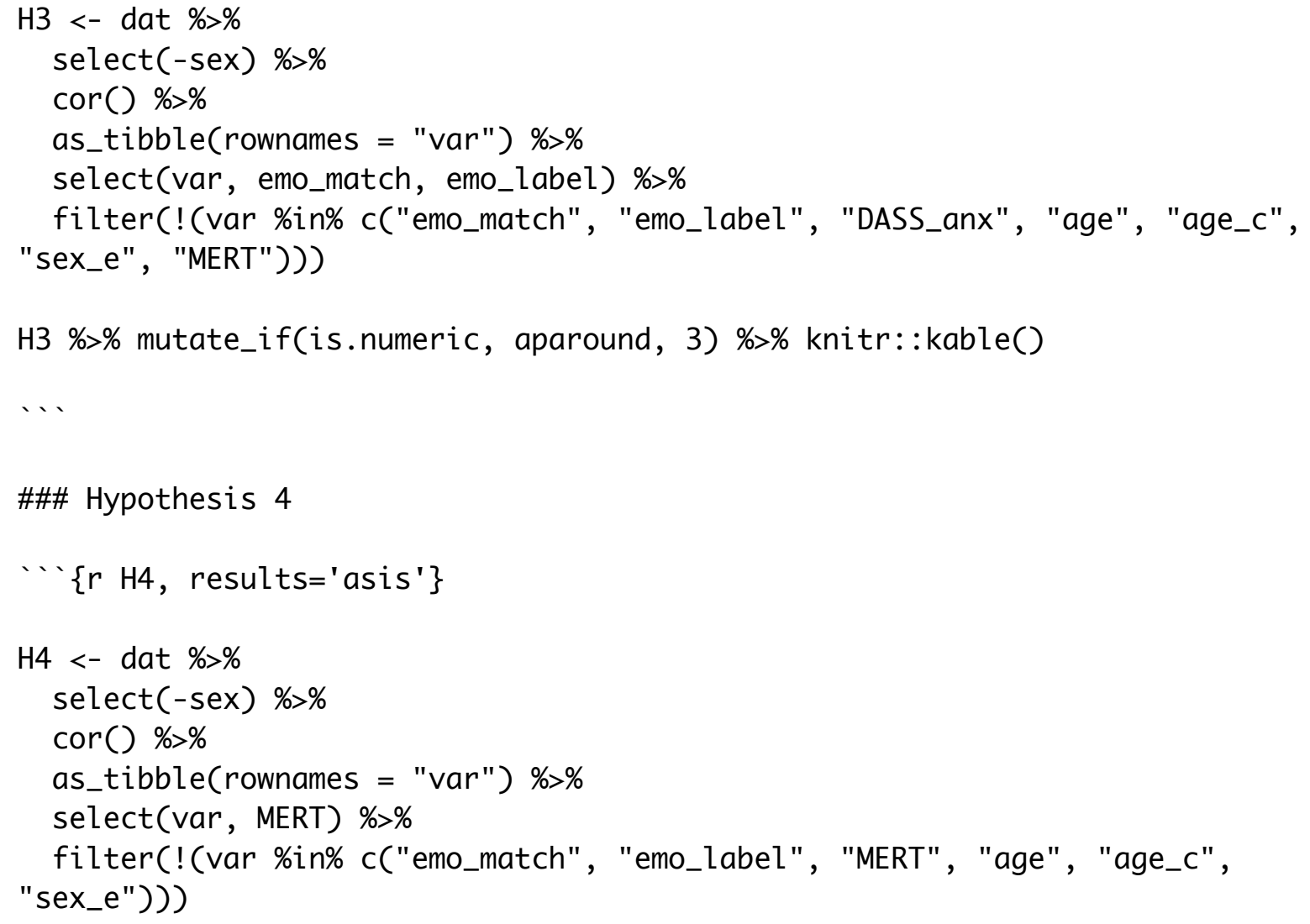

H4 \% \% mutate_if(is.numeric, aparound, 3) \% \% knitr::kable()

\#\# Robustness Checks

\#\#\# Truncated to Original Range

Truncate data to the original range from Table 1 in Palermo et al (2018).

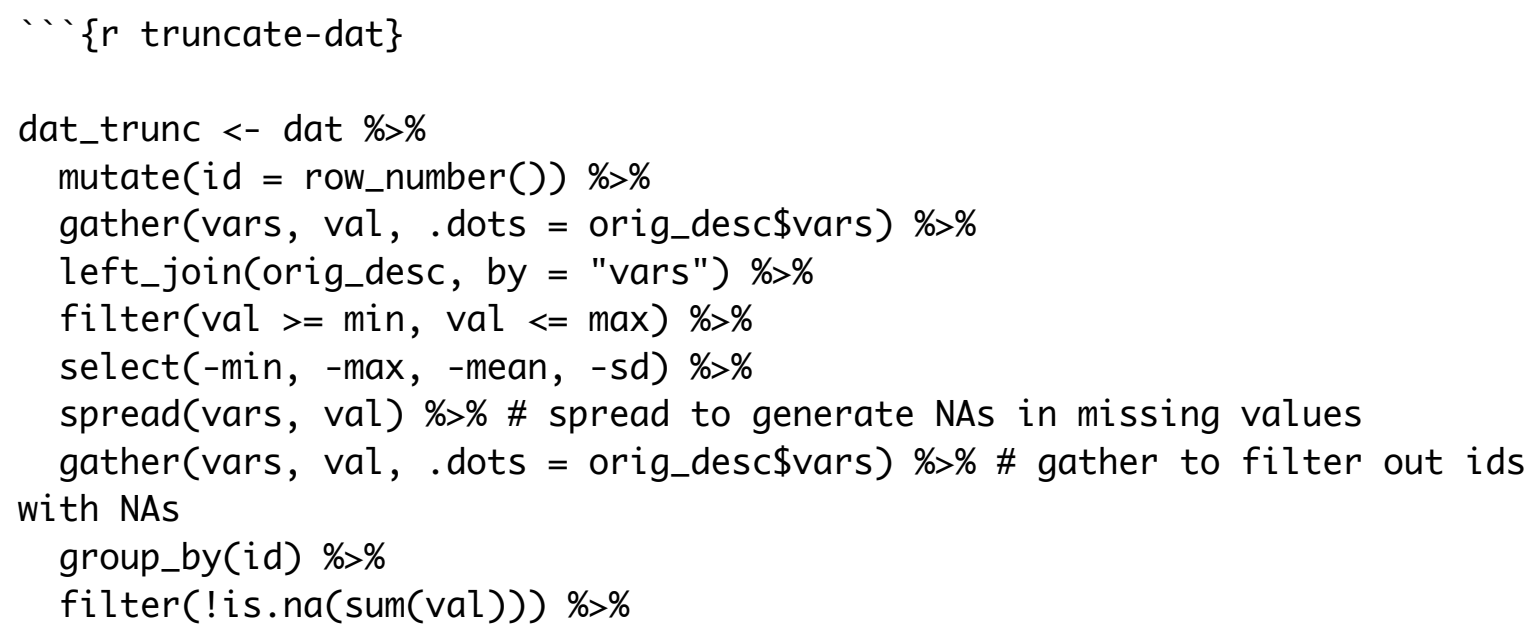




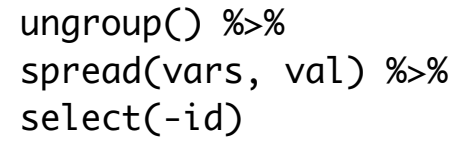

\#\#\#\# Hypothesis 1

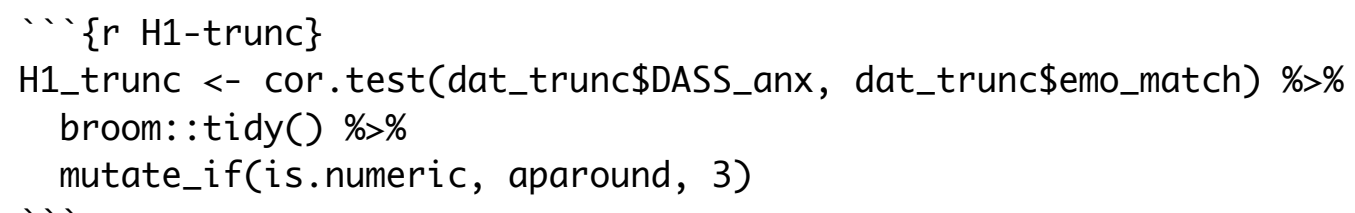

For data truncated to the original range from Palermo et al. (2018), scores on the emotion matching task and the DASS anxiety subscale were correlated

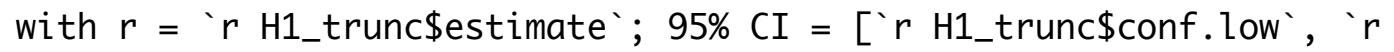
H1_trunc\$conf.high`], ' $r$ pval(H1_trunc\$p.value)`.

\#\#\#\# Hypothesis 2

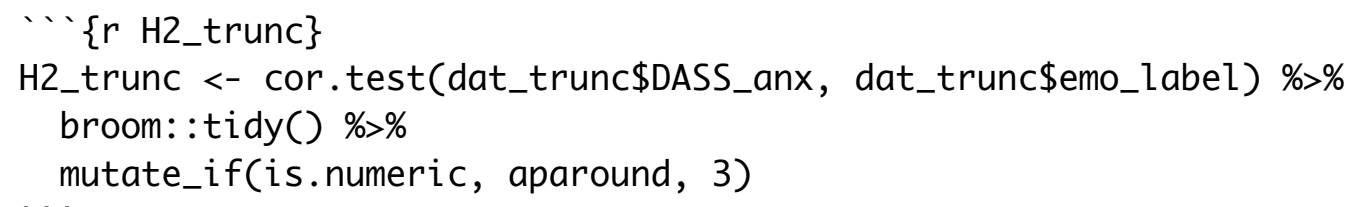

For data truncated to the original range from Palermo et al. (2018), scores on the emotion labeling task and the DASS anxiety subscale were correlated with $r={ }^{\prime} r$ H2_trunc $\$$ estimate`; 95\% CI $=$ [` $r$ H2_trunc $\$$ conf.low', ' $r$ H2_trunc\$conf.high`], ' $r$ pval(H2_trunc\$p.value)`.

\#\#\#\# Hypothesis 3

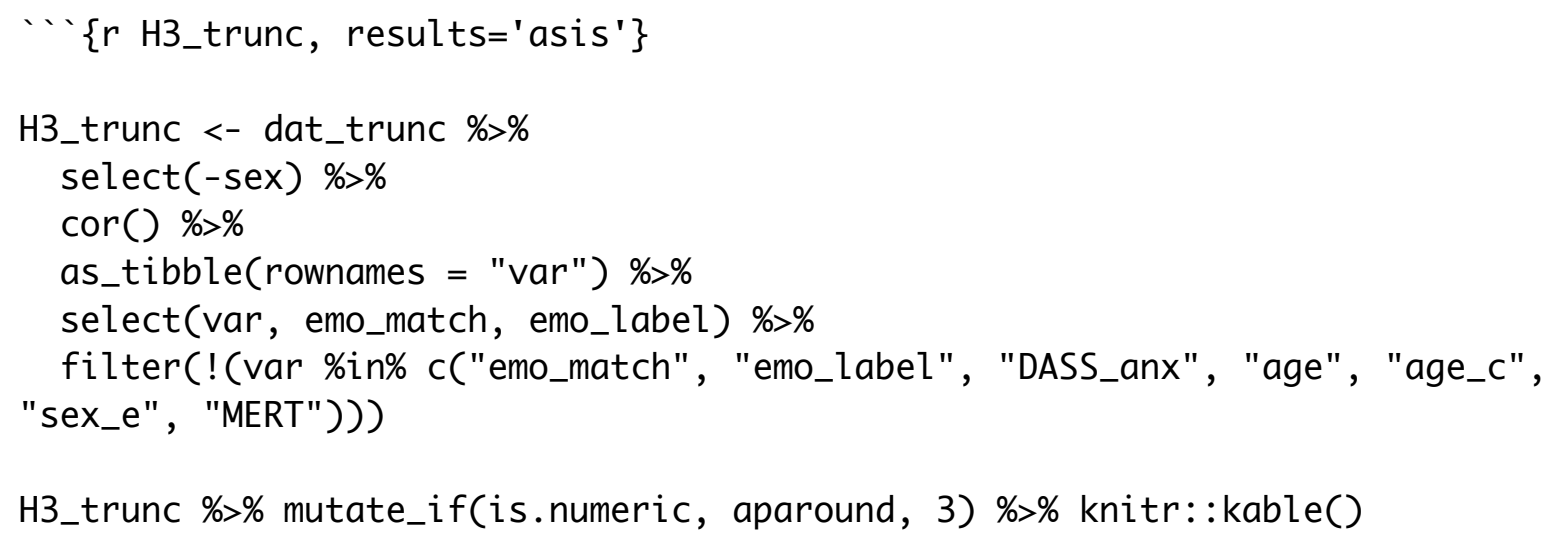

\#\#\#\# Hypothesis 4 
Controlling for sex, Scores on the DASS anxiety subscale negatively predicted scores on the emotion matching task

Cestimate $=$ ' $r$ rc_sex_stats\$estimate`;

95\% CI = [`r rc_sex_stats\$conf.low’, 'r rc_sex_stats\$conf.high`];

$t=' r\left(r c_{-}\right.$sex_stats $\$$ statistic $) ;$

'r pval(rc_sex_stats\$p.value)').

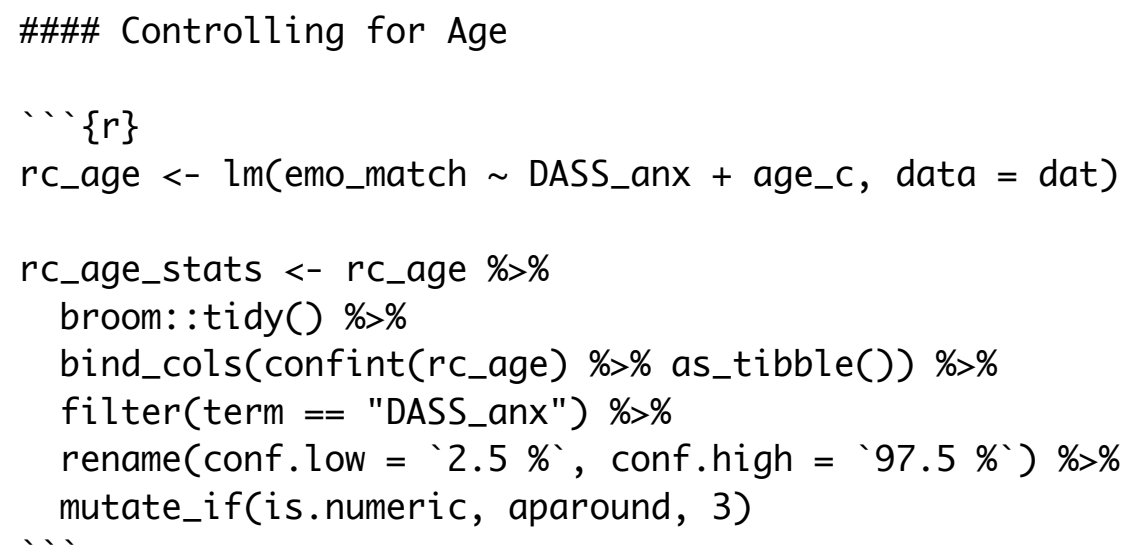

Controlling for age, Scores on the DASS anxiety subscale negatively predicted scores on the emotion matching task

(estimate = ' $r$ rc_age_stats\$estimate`;

95\% CI = [ $r$ rc_age_stats\$conf.low`, 'r rc_age_stats\$conf.high`];

$t={ }^{\prime} r\left(r c \_a g e \_s t a t s \$ s t a t i s t i c\right) ;$

'r pval(rc_age_stats\$p.value)').

\#\#\#\# Controlling for Sex and Age

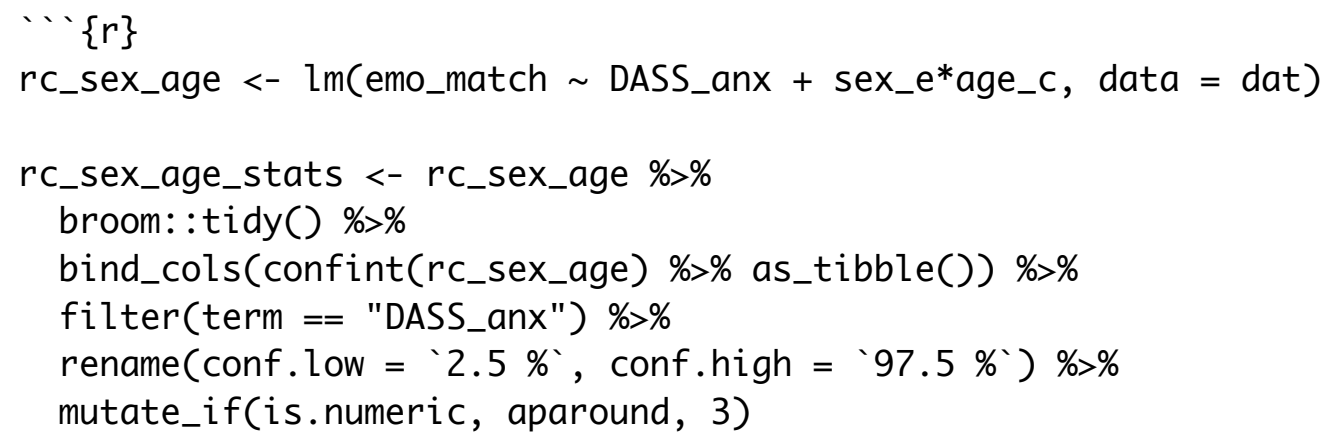

Controlling for sex, age and their interaction, Scores on the DASS anxiety subscale negatively predicted scores on the emotion matching task (estimate $=$ ' $r$ rc_sex_age_stats\$estimate ';

95\% CI = [`r rc_sex_age_stats\$conf. low`, 'r rc_sex_age_stats\$conf.high` $]$; 


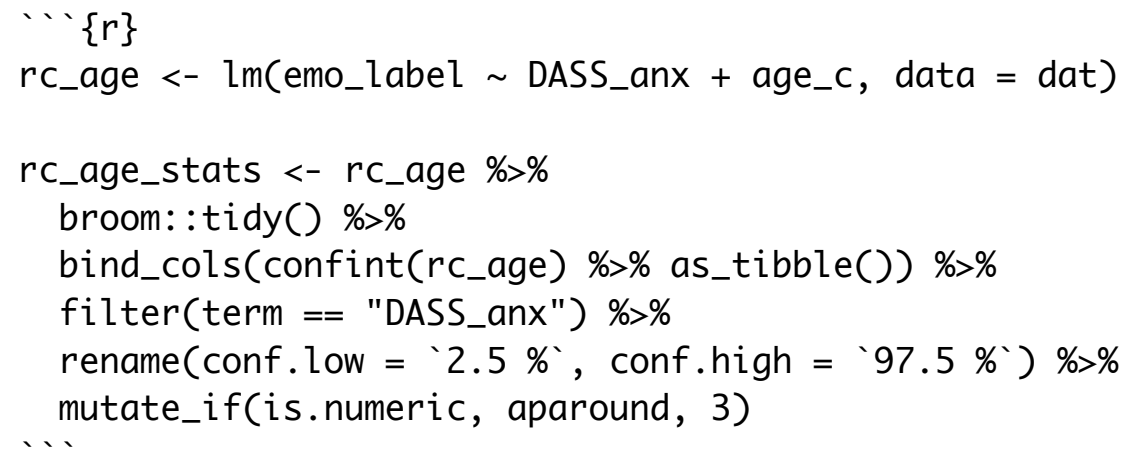

Controlling for sex, age and their interaction, Scores on the DASS anxiety subscale negatively predicted scores on the emotion labelling task (estimate = 'r rc_sex_age_stats\$estimate`;

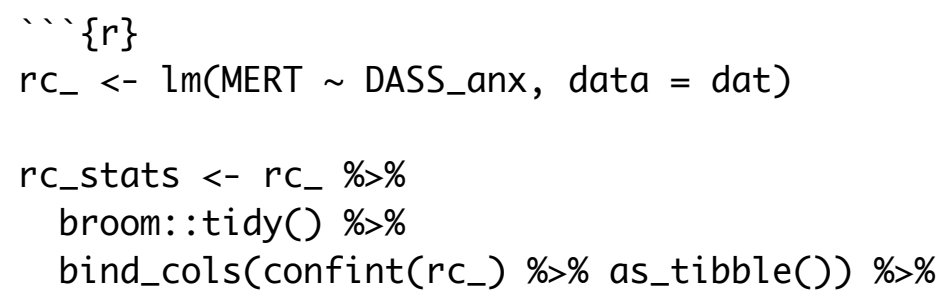




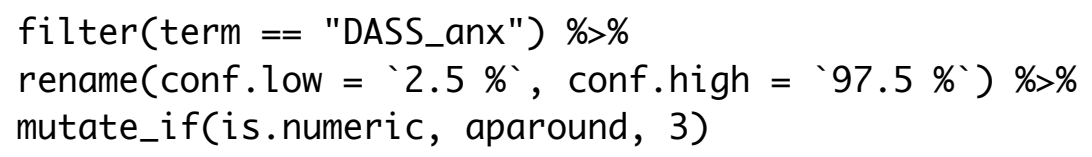

Scores on the DASS anxiety subscale negatively predicted scores on the MERT (estimate $=$ ' $r$ rc_stats\$estimate ';

Controlling for sex, Scores on the DASS anxiety subscale negatively predicted scores on the MERT

(estimate $=$ ' $r$ rc_sex_stats\$estimate`;

95\% CI = [`r rc_sex_stats\$conf.low`, 'r rc_sex_stats $\$$ conf.high $]$;

$t=' r\left(r c_{-}\right.$sex_stats $\$$ statistic $) ;$

'r pval(rc_sex_stats\$p.value)').

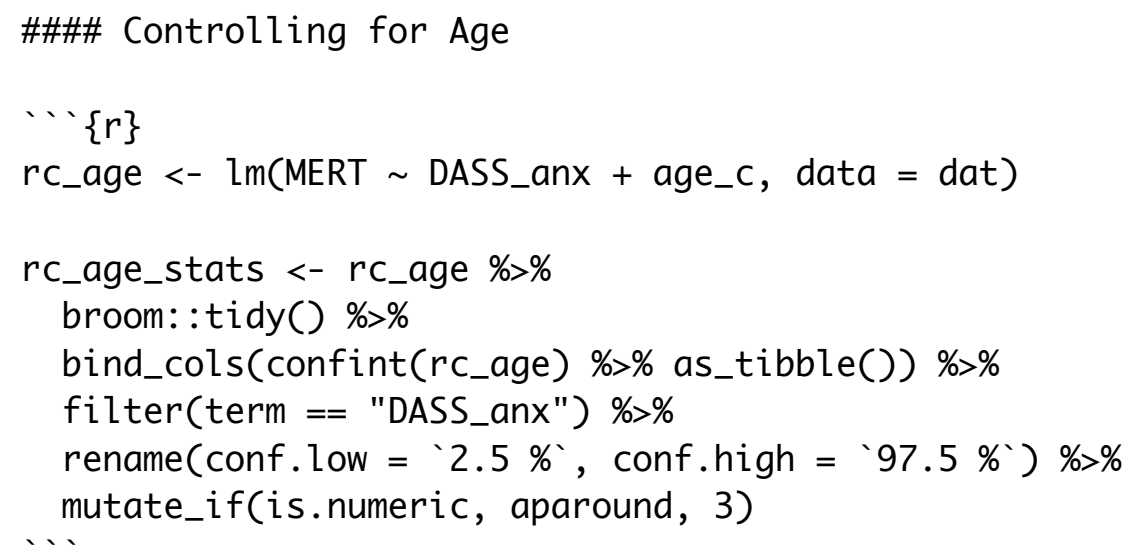

Controlling for age, Scores on the DASS anxiety subscale negatively predicted scores on the MERT 


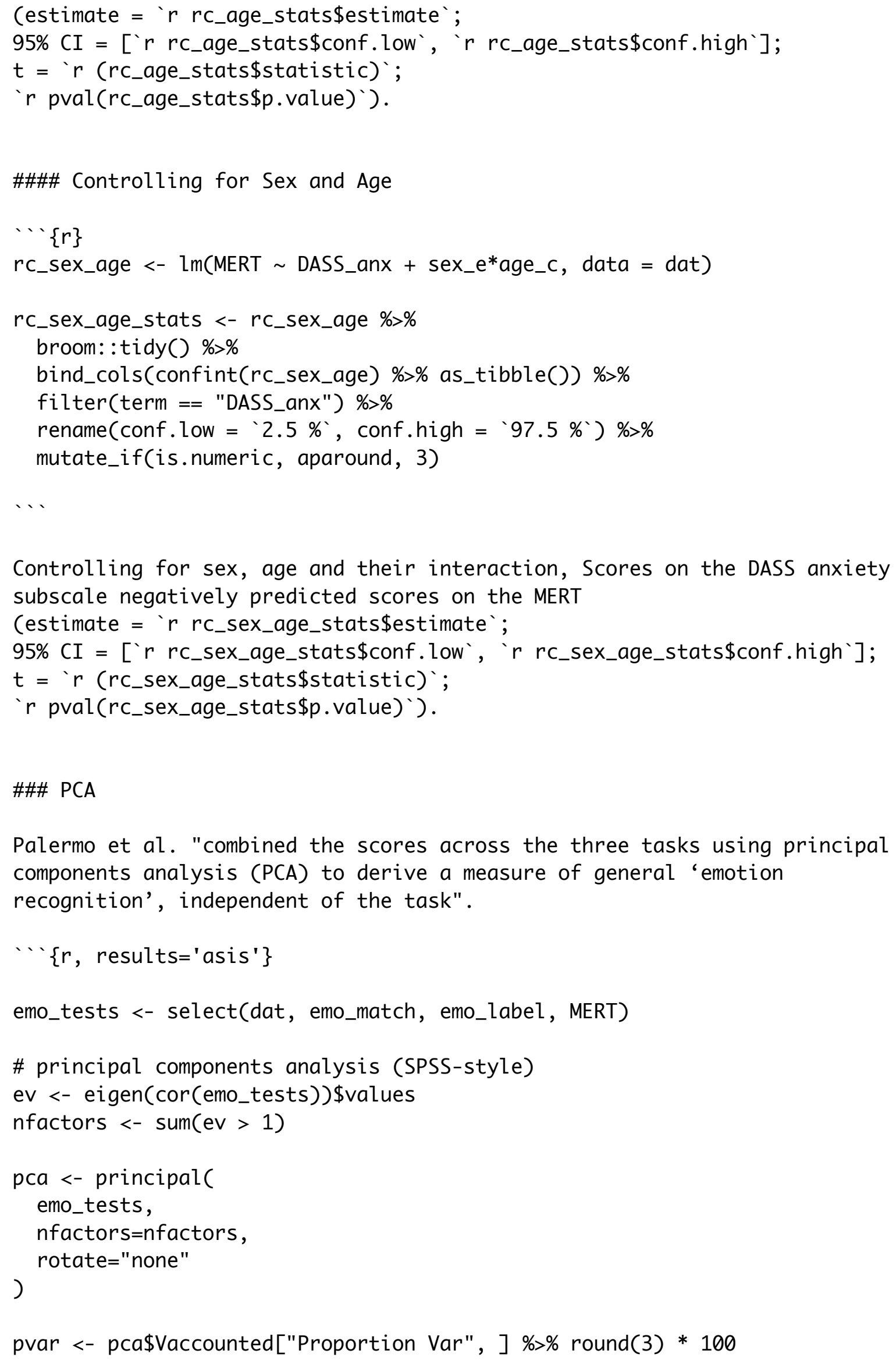

Controlling for sex, age and their interaction, Scores on the DASS anxiety subscale negatively predicted scores on the MERT (estimate $=$ ' $r$ rc_sex_age_stats\$estimate' ;

Palermo et al. "combined the scores across the three tasks using principal components analysis (PCA) to derive a measure of general 'emotion recognition', independent of the task".

$\cdots\left\{r\right.$, results $=^{\prime}$ asis' $\}$

emo_tests <- select(dat, emo_match, emo_label, MERT)

\# principal components analysis (SPSS-style)

ev <- eigen(cor(emo_tests)) \$values

nfactors <- $\operatorname{sum}(\mathrm{ev}>1)$

pca <- principal

emo_tests,

nfactors=nfactors, rotate $="$ none" 


$$
\begin{aligned}
& \text { unclass (pca\$loadings) } \%>\% \\
& \text { as.data.frame() } \%>\% \\
& \text { rownames_to_column() } \%>\% \\
& \text { knitr::kable(digits = } 2 \text { ) }
\end{aligned}
$$

The proportion of variance explained by this principal component was ' $r$ pvar $\%$.

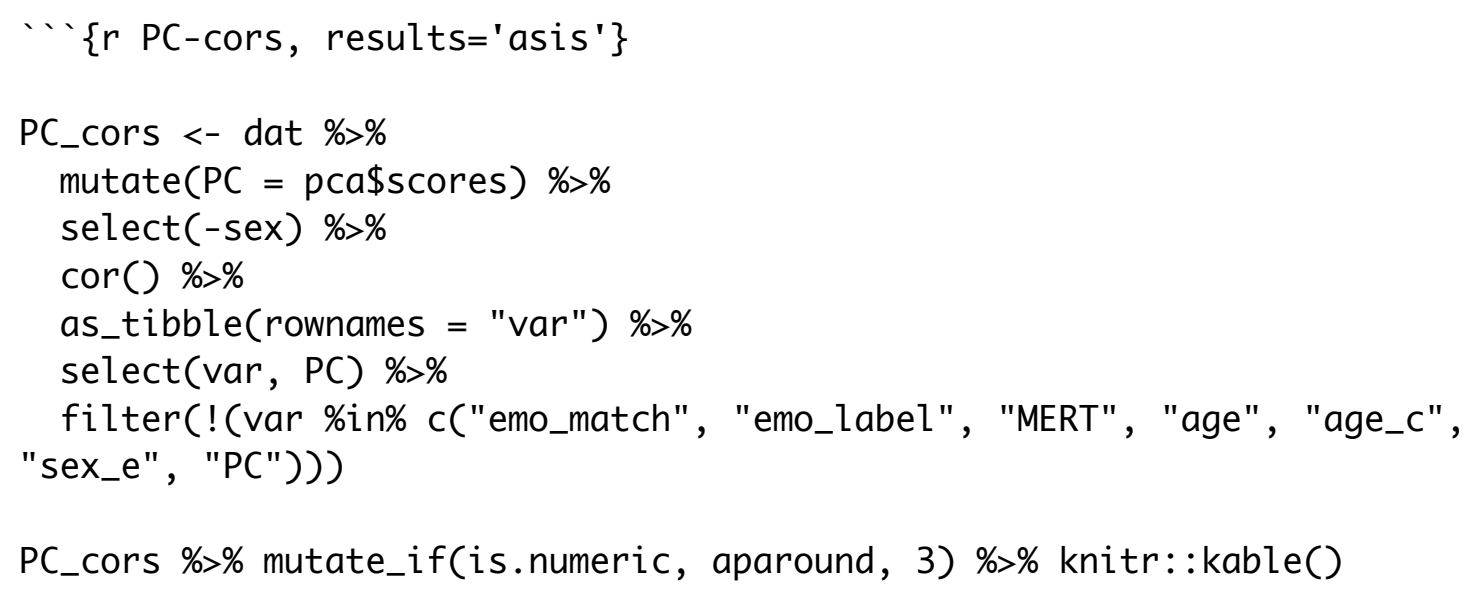


- Sample Size

- Analysis

- Simulate data

- Hypothesis 1

- Hypothesis 2

- Hypothesis 3

- Hypothesis 4

- Robustness Checks

- Truncated to Original Range

- Emotion Matching

- Emotion Labeling

- MERT

- PCA

\section{Sample Size}

Sample size is set at $n=160$, following the small telescopes approach, which sets replication sample size at 2.5 times the original sample size ( $n=63$ ). With alpha $=.05$, this design has $80 \%$ power for two-tailed tests to detect correlations as small as $|r|=0.219$.

\section{Analysis}

\section{Simulate data}

Descriptive data are taken from from Palermo et al (2018) with correlation structure from correlations reported in Palermo et al. (2018) or set to $\mathrm{r}=$ 0.5 for all depression/anxiety measures (as unreported in Palermo).

\begin{tabular}{|c|c|c|c|c|c|c|c|c|c|c|c|c|c|c|}
\hline var & MERT & emo_match & emo_label & DASS_dep & DASS_anx & PANAS_p & PANAS_n & $\mathbf{A Q}$ & EQ & BES_aff & BES_cog & age & sex_e & $\mathbf{a}$ \\
\hline MERT & 1.000 & 0.545 & 0.550 & -0.126 & -0.321 & -0.330 & -0.151 & -0.105 & -0.029 & -0.133 & 0.047 & 0.059 & 0.052 & ( \\
\hline emo_match & 0.545 & 1.000 & 0.581 & -0.188 & -0.315 & -0.213 & -0.298 & -0.098 & -0.029 & -0.101 & 0.055 & 0.039 & 0.006 & ( \\
\hline emo_label & 0.550 & 0.581 & 1.000 & -0.167 & -0.305 & -0.188 & -0.137 & -0.170 & -0.057 & -0.037 & 0.040 & -0.028 & 0.046 & $-c$ \\
\hline DASS_dep & -0.126 & -0.188 & -0.167 & 1.000 & 0.578 & 0.489 & 0.542 & 0.502 & 0.560 & 0.459 & 0.484 & 0.048 & 0.008 & ( \\
\hline DASS_anx & -0.321 & -0.315 & -0.305 & 0.578 & 1.000 & 0.617 & 0.493 & 0.514 & 0.553 & 0.573 & 0.523 & 0.025 & 0.129 & ( \\
\hline PANAS_p & -0.330 & -0.213 & -0.188 & 0.489 & 0.617 & 1.000 & 0.505 & 0.548 & 0.544 & 0.483 & 0.522 & 0.058 & 0.021 & ( \\
\hline PANAS_n & -0.151 & -0.298 & -0.137 & 0.542 & 0.493 & 0.505 & 1.000 & 0.540 & 0.571 & 0.562 & 0.536 & 0.087 & 0.072 & ( \\
\hline$A Q$ & -0.105 & -0.098 & -0.170 & 0.502 & 0.514 & 0.548 & 0.540 & 1.000 & 0.599 & 0.560 & 0.627 & 0.134 & 0.137 & ( \\
\hline $\mathrm{EQ}$ & -0.029 & -0.029 & -0.057 & 0.560 & 0.553 & 0.544 & 0.571 & 0.599 & 1.000 & 0.550 & 0.611 & 0.128 & 0.133 & ( \\
\hline BES_aff & -0.133 & -0.101 & -0.037 & 0.459 & 0.573 & 0.483 & 0.562 & 0.560 & 0.550 & 1.000 & 0.572 & 0.060 & 0.150 & ( \\
\hline BES_cog & 0.047 & 0.055 & 0.040 & 0.484 & 0.523 & 0.522 & 0.536 & 0.627 & 0.611 & 0.572 & 1.000 & 0.124 & 0.193 & ( \\
\hline age & 0.059 & 0.039 & -0.028 & 0.048 & 0.025 & 0.058 & 0.087 & 0.134 & 0.128 & 0.060 & 0.124 & 1.000 & -0.075 & - \\
\hline sex_e & 0.052 & 0.006 & 0.046 & 0.008 & 0.129 & 0.021 & 0.072 & 0.137 & 0.133 & 0.150 & 0.193 & -0.075 & 1.000 & $-c$ \\
\hline age_c & 0.059 & 0.039 & -0.028 & 0.048 & 0.025 & 0.058 & 0.087 & 0.134 & 0.128 & 0.060 & 0.124 & 1.000 & -0.075 & 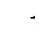 \\
\hline
\end{tabular}

\section{Hypothesis 1}

Scores on the emotion matching task and the DASS anxiety subscale were correlated with $r=-0.315 ; 95 \% \mathrm{Cl}=[-0.448,-0.168], \mathrm{p}<.001$.

\section{Hypothesis 2}

\section{Hypothesis 3}




\begin{tabular}{lll}
\hline DASS_dep & -0.188 & -0.167 \\
\hline PANAS_p & -0.213 & -0.188 \\
\hline PANAS_n & -0.137 \\
\hline AQ & -0.298 & -0.098 \\
\hline EQ & -0.057 \\
\hline BES_aff & -0.029 \\
\hline BES_cog & -0.101 & -0.037 \\
\hline
\end{tabular}

\section{Hypothesis 4}

\begin{tabular}{ll} 
Code & MERT \\
\hline var & -0.126 \\
\hline DASS_dep & -0.321 \\
\hline DASS_anx & -0.330 \\
\hline PANAS_p & -0.151 \\
\hline PANAS_n & -0.105 \\
\hline AQ & -0.029 \\
\hline EQ & -0.133 \\
\hline BES_aff & 0.047 \\
\hline BES_cog &
\end{tabular}

\section{Robustness Checks}

\section{Truncated to Original Range}

Truncate data to the original range from Table 1 in Palermo et al (2018).

Hypothesis 1

For data truncated to the original range from Palermo et al. (2018), scores on the emotion matching task and the DASS anxiety subscale were correlated with $r=-0.306 ; 95 \% \mathrm{Cl}=[-0.468,-0.125], \mathrm{p}=0.001$.

\section{Hypothesis 2}

For data truncated to the original range from Palermo et al. (2018), scores on the emotion labeling task and the DASS anxiety subscale were correlated with $r=-0.157 ; 95 \% \mathrm{Cl}=[-0.335,0.032], \mathrm{p}=0.104$.

Hypothesis 3

var emo_match emo_label

$A Q$ 0.019 0.065

BES_aff 0.073 0.235

BES_cog

0.233

0.300

DASS_dep

$-0.147$

$-0.059$

EQ

0.095

0.199

PANAS_n

$-0.182$

0.104

PANAS_p

$-0.134$

$-0.011$

\section{Hypothesis 4}

var 


$\begin{array}{ll}\text { PANAS_n } & -0.151 \\ \text { AQ } & -0.105 \\ \text { EQ } & -0.029 \\ \text { BES_aff } & -0.133 \\ \text { BES_cog } & 0.047\end{array}$

\section{Emotion Matching}

Scores on the DASS anxiety subscale negatively predicted scores on the emotion matching task $(\mathrm{estimate}=-0.862 ; 95 \% \mathrm{Cl}=[-1.27,-0.453]$; $\mathrm{t}=$ $-4.167 ; p<.001)$.

\section{Controlling for Sex}

Controlling for sex, Scores on the DASS anxiety subscale negatively predicted scores on the emotion matching task (estimate = -0.879 ; $95 \% \mathrm{Cl}=$ $[-1.291,-0.466] ; \mathrm{t}=-4.205 ; \mathrm{p}<.001)$.

\section{Controlling for Age}

Controlling for age, Scores on the DASS anxiety subscale negatively predicted scores on the emotion matching task $($ estimate $=-0.865 ; 95 \% \mathrm{Cl}=$ $[-1.275,-0.456] ; \mathrm{t}=-4.173 ; \mathrm{p}<.001)$.

\section{Controlling for Sex and Age}

Controlling for sex, age and their interaction, Scores on the DASS anxiety subscale negatively predicted scores on the emotion matching task (estimate $=-0.893 ; 95 \% \mathrm{Cl}=[-1.307,-0.478] ; \mathrm{t}=-4.257 ; \mathrm{p}<.001)$.

\section{Emotion Labeling}

Scores on the DASS anxiety subscale negatively predicted scores on the emotion labelling task $(\mathrm{estimate}=-0.611 ; 95 \% \mathrm{Cl}=[-0.91,-0.311] ; \mathrm{t}=$ $-4.03 ; p<.001)$.

\section{Controlling for Sex}

Controlling for sex, Scores on the DASS anxiety subscale negatively predicted scores on the emotion labelling task $($ estimate $=-0.633$; $95 \% \mathrm{Cl}=$ $[-0.935,-0.331] ; \mathrm{t}=-4.146 ; \mathrm{p}<.001)$.

\section{Controlling for Age}

Controlling for age, Scores on the DASS anxiety subscale negatively predicted scores on the emotion labelling task (estimate $=-0.61 ; 95 \% \mathrm{Cl}=$ $[-0.91,-0.309] ; \mathrm{t}=-4.01 ; \mathrm{p}<.001)$.

\section{Controlling for Sex and Age}

Controlling for sex, age and their interaction, Scores on the DASS anxiety subscale negatively predicted scores on the emotion labelling task (estimate $=-0.634 ; 95 \% \mathrm{Cl}=[-0.938,-0.33] ; \mathrm{t}=-4.119 ; \mathrm{p}<.001)$.

\section{MERT}

Scores on the DASS anxiety subscale negatively predicted scores on the MERT (estimate $=-0.683 ; 95 \% \mathrm{Cl}=[-0.999,-0.366] ; \mathrm{t}=-4.265 ; \mathrm{p}<$ .001).

\section{Controlling for Sex}

Controlling for sex, Scores on the DASS anxiety subscale negatively predicted scores on the MERT (estimate = $-0.708 ; 95 \% \mathrm{Cl}=[-1.027,-0.39] ; \mathrm{t}$ $=-4.398 ; p<.001)$

\section{Controlling for Age}

Controlling for age, Scores on the DASS anxiety subscale negatively predicted scores on the $\mathrm{MERT}(\mathrm{estimate}=-0.686 ; 95 \% \mathrm{Cl}=[-1.003,-0.37] ; \mathrm{t}$ $=-4.283 ; \mathrm{p}<.001)$.

\section{Controlling for Sex and Age}




\section{PCA}

Palermo et al. "combined the scores across the three tasks using principal components analysis (PCA) to derive a measure of general 'emotion recognition', independent of the task".

\begin{tabular}{ll} 
Code & PC1 \\
rowname & 0.84 \\
\hline emo_match & 0.85 \\
\hline MERT & 0.83
\end{tabular}

The proportion of variance explained by this principal component was $70.6 \%$.

$\begin{array}{ll}\text { DASS_dep } & -0.191 \\ \text { DASS_anx } & -0.373 \\ \text { PANAS_p } & -0.289 \\ \text { PANAS_n } & -0.233 \\ \text { AQ } & -0.148 \\ \text { EQ } & -0.046 \\ \text { BES_aff } & -0.107 \\ \text { BES_cog } & 0.057\end{array}$

